\title{
Non-Invasive Genotyping of Transgenic Mice: Comparison of Different Commercial Kits and Required Amounts
}

\author{
Melanie Hamann, Nikola Lange, Jagoda Kuschka and Angelika Richter \\ Institute of Pharmacology and Toxicology, Department of Veterinary Medicine, Freie Universität Berlin, Germany
}

\begin{abstract}
Summary
The usefulness of genetically modified mice is discussed critically. Nevertheless, their number has increased dramatically over the past years. A principle for the use these mice is the isolation of DNA to determine whether an individual carries the genetic modification or not. This "genotyping" is usually done by invasive tissue sampling, e.g. tail biopsies, which likely causes discomfort and pain to the animals and is therefore discussed in animal welfare regulations. Although non-invasive tissue sampling by stool samples was already described over 10 years ago, it is not commonly used so far.

In the present study we therefore tested the practicality of this method by the use of three commercial kits for genotyping and compared the results to those attained using tail biopsies.

Our data shows that DNA isolation from stool samples is practical, sensitive and effective. In addition, the possibility of repeated sampling represents a clear advantage to invasive genotyping. Therefore, this method represents a useful tool in the $3 R$ concept, since replacement of invasive tissue sampling refines the use of transgenic mice.
\end{abstract}

Keywords: tail biopsy, stool samples, faecal samples, genetically modified animals, genotyping

\section{Introduction}

Since their introduction in the 1980s, studies involving genetically modified rodents have increased dramatically although their usefulness in biomedical science is discussed critically (Buehr et al., 2003; Sauer et al., 2005, 2006). A basic necessity for the generation and use of these animals is the ability to distinguish mice that carry a disrupted gene or transgene from the wildtype animals. This so-called "genotyping" is routinely applied by using tail or ear biopsies or by collecting blood samples (Arras et al., 2007; Robinson et al., 2003; Schneider and Wolf, 2005). All these invasive methods are likely to cause discomfort and pain to the animals, although this fact has not been investigated extensively so far (Arras et al., 2007; Buehr et al., 2003; Robinson et al., 2003; Schneider and Wolf, 2005). Furthermore, the value of anaesthesia and analgesia to avoid pain and stress caused by these invasive methods is discussed controversially (Arras et al., 2007; Robinson et al., 2003).
Abbreviations
bp basepair
DNA deoxyribonucleic acid
GAG nucleonic acid triplet guanine-adenine-guanine
PCR polymerase chain reaction

Therefore, non-invasive methods are required to replace invasive methods and to reduce discomfort. During the past years, several methods of non-invasive genotyping were proposed, including sampling of saliva by oral swabs or pipettes, the use of hair samples or samples of rectum cells acquired by rectal swabs (for review: Pinkert, 2003; Robinson et al., 2003; Schneider and Wolf, 2005). Although all these methods avoid invasive tissue sampling, they do require restraining of the animals and therefore may cause discomfort. In addition, some methodological problems like the need of nested PCR in saliva samples of younger animals or the higher incidence of carrying over contaminations were suggested (Broome et al., 1999; Irwin et al., 1996; Robinson et al., 2003; Schneider and Wolf, 2005; Truett et al., 2000). Therefore, the use of these methods is limited and they have no widespread acceptance so far.

Previously, three studies described the genotyping of mice using stool analysis, the only non-invasive genotyping method that does not require restraint and therefore avoids tissue sampling and handling stress (Broome et al., 1999; Kalippke et al., 2009; Murgatroyd et al., 2006). This method was initially described 10 years ago (Broome et al., 1999), but is still not in common use. Up to now, it is not clear whether the infrequent use of this method is based in practical reasons, a widespread lack of knowledge of its existence (Schneider and Wolf, 2005)

Received $16^{\text {th }}$ April 2010, received in revised form and accepted for publication $28^{\text {th }}$ July 2010 
or maybe the preference of keeping to traditional methods and avoiding uncomfortable testing of new insights.

Although the collection of faecal samples from mice can be easily done during the routine change of home cages, the uncommon use of this method may be related to some difficulties. First of all, stool samples typically contain many substances like nucleases and other enzymes that can degrade DNA and inhibit downstream enzymatic reactions, complicating the isolation and purification of genomic DNA. To ensure removal of these substances, commercial kits for the extraction of DNA from stool specimens include inhibitors wich efficiently adsorb these substances. However, these kits were developed for the extraction of human DNA or of bacterial DNA from human faecal samples, but not for genotyping of small laboratory rodents. Therefore, large amounts of stool specimens are required in the protocols for the commercial kits. This fact is often stated as a disadvantage of this method, because these amounts can only be sampled by multiple handling of mice or a long-term stay in clean and empty cages, which is more time-consuming than tail or ear biopsies.

In order to clarify the practicality of non-invasive genotyping by using murine stool pellets, we compared the results of three different commercial kits developed for the purification and isolation of DNA from human stool specimens with the results of a commercial kit developed for genotyping of tissue from tail biopsies. Furthermore, we compared the results of larger amounts of stool samples, as recommended by the kits' producers, with those of smaller samples. This study follows the recommendation mentioned in the sixth report of the BVAAWF/FRAME/ RSPCA/UFAW joint working group on refinement to further investigate the use of faecal samples as non-invasive sources of DNA for genotyping (Robinson et al., 2003).

\section{Animals}

Breeding pairs of transgenic DYT1 mice were kindly provided by Pullani Shashidharan, Mount Sinai School of Medicine, New York, USA. The human gene DYT1 represents a defective gene that leads to a 3 basepair (GAG) deletion resulting in a loss of a glutamic acid residue $(\Delta \mathrm{E})$ in the protein torsin $\mathrm{A}$ (Breakefield et al., 2008; Tanabe et al., 2009). The gene defect causes earlyonset torsion dystonia, a common movement disorder (Breakefield et al., 2008; Tanabe et al., 2009). The mice, which were generated on a C57BL/6J background, were free of all viral, bacterial and parasitic pathogens listed in the Federation of European Laboratory Animal Associations (FELASA) recommendations (Nicklas et al., 2002). They were kept in type 3 opentop makrolon cages $\left(420 \times 265 \times 150 \mathrm{~mm}\right.$, floor area $825 \mathrm{~cm}^{2}$, Ehret, Wandlitz, Germany) with dust-free wooden embedding (ssniff ${ }^{\circledR}$ Lignocell 3-4 S, ssniff Spezialdiäten GmbH, Soest, Germany). A maximum of three mice were kept together in one cage. Cages were enriched with cellulose and cardboard boxes as nesting material as well as sunflower seeds in the embedding to satisfy seeking behaviour. Mice were fed a pelleted mouse diet (ssniff ${ }^{\circledR}$ EF R/M-H 10 mm, ssniff Spezialdiäten GmbH, Soest, Germany) ad libitum and had free access to water. The light/dark cycle in the room consisted of $12 / 12 \mathrm{~h}$ with artificial light ( $\sim 100$ Lux in the cage). The room temperature was 23 $\pm 1^{\circ} \mathrm{C}$, with a relative humidity of $50 \pm 10 \%$. Breeding and husbandry as well as the execution of tail biopsies were approved by the German Animal Welfare Agency (registration numbers ZH 5 and $\mathrm{O}$ 0119/07).

\section{Materials and methods}

\section{Sampling of stool pellets}

Out of 45 mice at an age of 21 days, which were routinely genotyped by DNA extraction from stool samples with the QIAamp ${ }^{\circledR}$ DNA Stool Mini Kit (Qiagen, Berlin, Germany) in our institute, three transgenic and three wildtype control mice were used exemplarily in the study presented here for the comparison of the three commercial DNA extraction kits described below. Each mouse was placed in an empty, clean and sterile makrolon standard cage. This procedure can be easily combined with the routine change of home cages. Usually, up to three stool droplets (each of $\sim 20 \mathrm{mg}$ ) were excreted within 1-2 minutes after placing 21 days old mice in a new cage. The stool droplets were collected with sterile tweezers, placed in a DNA extraction tube and stored at $-80^{\circ} \mathrm{C}$ until DNA isolation and purification. To collect an amount of 180-200 mg stool specimen per mouse, recommended by the manufacturers of commercial kits (see below), this procedure was performed three to four times with each mouse. Although a single longer stay ( $\sim 30-60$ minutes $)$ would also have enabled sampling the higher amount of stool, we preferred multiple short-time sampling to avoid restriction of food and water for animals.

In addition, five 10 day old mice were placed singularly in an empty, clean and sterile makrolon standard cage for 5 minutes to investigate whether these young animals already produce a sufficient amount of stool samples.

\section{Tail biopsy}

One transgenic DYT1 and one wildtype control mouse were restrained by the skin of the neck, a small segment $(<5 \mathrm{~mm})$ of the tail tip was cut off with a sharp surgical scissor and the tail segment was placed immediately in a DNA extraction tube and stored at $-80^{\circ} \mathrm{C}$ until DNA isolation and purification. No treatment for haemostasis was required as bleeding was minimal. To avoid infection, an iodine solution was applied to the wound at the tail tip. After this procedure, the mice were immediately returned to their home cages.

\section{Isolation and purification of genomic DNA}

For the isolation and purification of genomic DNA from stool samples, we used three different commercial kits: a) QIAamp ${ }^{\circledR}$ DNA Stool Mini Kit (Qiagen, Berlin, Germany), b) innuPREP ${ }^{\circledR}$ Stool DNA Kit (Analytik Jena, Jena, Germany) and c) EURx ${ }^{\circledR}$ Gene Matrix Stool DNA Kit (Roboklon GmbH, Berlin, Germany). The required amounts of stool samples, recommended by the producers, were for kit a) $180-220 \mathrm{mg}$, for kit b) $200-400 \mathrm{mg}$ and for kit c) $200 \mathrm{mg}$. In a first step, we tested the applicability of the kits for the detection of the transgene in mice in accord- 
ance with the protocol provided by the manufacturers. Here, we used the recommended amounts, i.e. 9-10 stool droplets per mouse were processed. In a second step, we used only 2 stool droplets ( 40-45 mg) for all three kits but then proceeded with the isolation and purification of genomic DNA in accordance with the protocols provided by the producers.

For the isolation and purification of genomic DNA from tail biopsies we used the Invisorb ${ }^{\circledR}$ Spin Tissue Mini Kit (Invitek, Berlin, Germany). Isolation and purification were performed in accordance with the producer's manual.

Fig. 1:

a) Example of genotyping via PCR on DNA isolated from tail biopsy $(E, G)$ or stool specimens $(A, C)$ followed by digestion of the $\mathrm{PCR}$ product with BseR1 restriction enzyme (tail biopsy: $\mathrm{F}, \mathrm{H}$; stool specimens: B, D) using the "QIAamp ${ }^{\circledR}$ DNA Stool Mini Kit" (Qiagen, Berlin, Germany) for analysis of stool droplets or the "Invisorb ${ }^{\circledR}$ Spin Tissue Mini Kit" (Invitek, Berlin, Germany). PCR amplification of $\triangle \mathrm{E}$-torsinA cDNA yielded a $213 \mathrm{bp}$ DNA product $(\mathrm{C}, \mathrm{E})$. Digestion of $\mathrm{PCR}$ amplified product from $\triangle \mathrm{E}$-torsinA CDNA yielded DNA fragments of $95 \mathrm{bp}$ and $118 \mathrm{bp}(\mathrm{D}, \mathrm{F})$ owing to the loss of a BseR1 restriction site. As shown in the figure, a very small amount of stool specimen ( $47 \mathrm{mg}=2$ stool pellets) was sufficient to detect positive DNA products in PCR amplification and digestion $(D)$, comparable to the tail biopsy $(E, F)$. The PCR products from the tail biopsies represent our positive $(E, F)$ and negative $(Q, R)$ control, since they were extracted from animals with a known genotype.

b) Example of genotyping via PCR on DNA isolated from stool specimens followed by digestion of the PCR product with BseR1 restriction enzyme using the EURx ${ }^{\circledR}$ Gene Matrix Stool DNA kit (Roboklon GmbH, Berlin, Germany) (G-L) and the innuPREP ${ }^{\circledR}$ Stool DNA kit (Analytik Jena, Jena, Germany). The signal of a high amount of stool specimen as proposed by the companies producing the kits (e.g. G, H) was comparable to that of tail biopsies (Fig. 1a: E, F). There were also no prominent differences between a small amount (G, $\mathrm{H}: 43 \mathrm{mg}=2$ stool droplets) and a higher amount (I, J: $132 \mathrm{mg}=6$ stool droplets) of stool specimen in the genotyping using the EURx ${ }^{\circledR}$ Gene Matrix Stool DNA kit. The innuPREP $B$ Stool DNA kit was also able to detect the PCR amplified and digested $\triangle E$-torsinA CDNA product using a small amount of stool specimen ( $\mathrm{M}, \mathrm{N}: 43 \mathrm{mg}=2$ stool droplets). $\mathrm{K}$ and $\mathrm{L}$ represent negative samples tested with the $E U R x^{\circledR}$ Gene Matrix Stool DNA kit, O and $\mathrm{P}$ a negative sample verified by the innuPREP ${ }^{\circledR}$ Stool DNA kit. For further explanations see legend to Fig. 1a).

\section{Genotype analysis}

Polymerase chain reaction (PCR) was performed on genomic DNA isolated from stool droplets or tail biopsies in accordance with previous descriptions of Shashidharan et al. (2005). For the analysis of the defective gene, DYT1, we used the following primer set: sense primer 5'-CCTGGAATACAAACACCTA-3' and antisense primer 5'-CAGTGACTCCGGCTGCCAATC-3'. The PCR cocktail (50 $\mu 1 /$ sample) contained $5 \mu 1$ Taq-Buffer (10x), $1 \mu 1 \mathrm{dNTP}$ mixture, $0.5 \mu 1$ sense primer, $0.5 \mu 1$ antisense primer, the calculated amount of genomic DNA of the sample

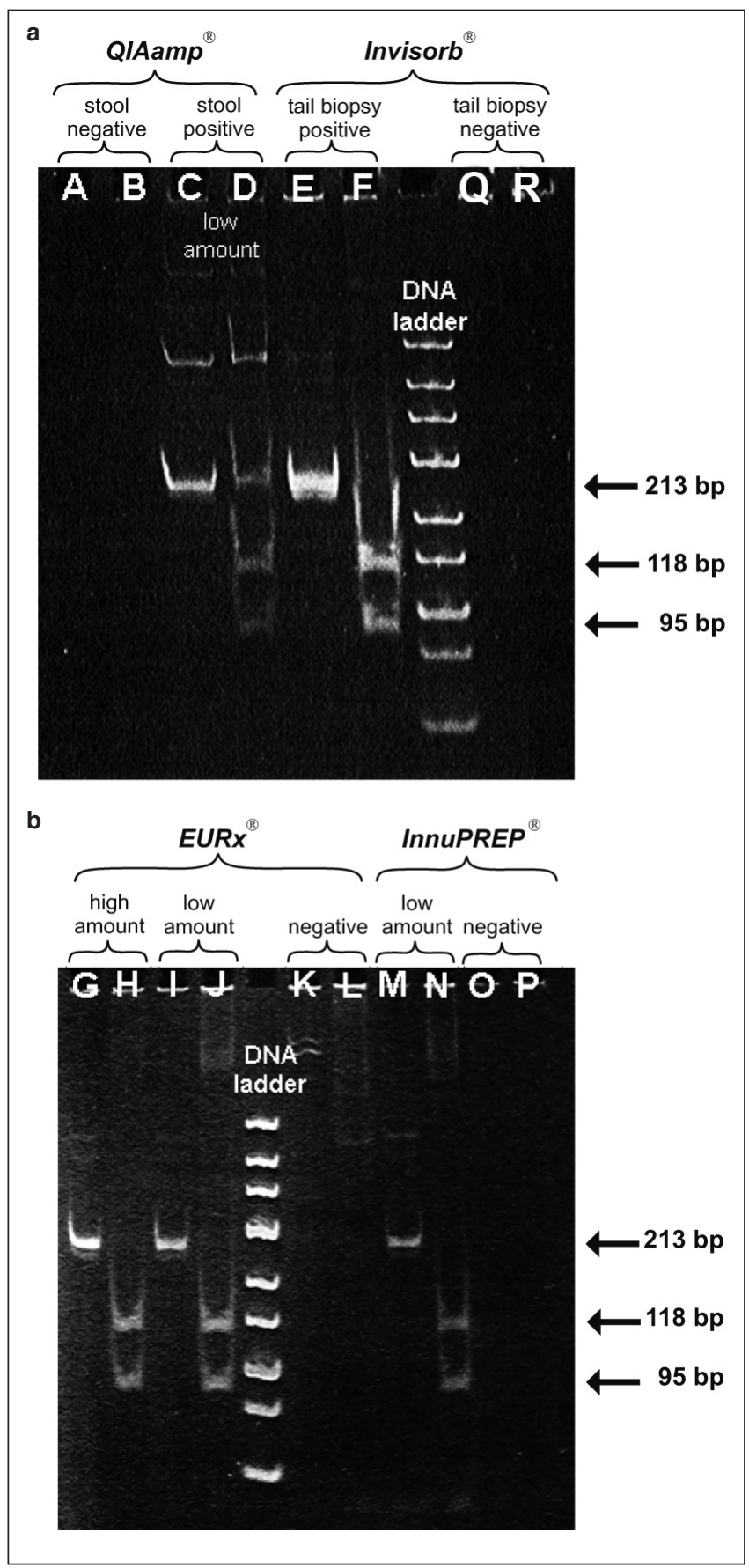


and an adequate amount of $\mathrm{dH}_{2} \mathrm{O}$ to fill up to the total volume of $50 \mu 1$. In a first step, TaqDNApolymerse (PEQLAB, Erlangen, Germany) was used to amplify the DNA under the following conditions: $94^{\circ} \mathrm{C}$ for $1 \mathrm{~min}$ (initial denaturation), followed by 30 cycles of $94^{\circ} \mathrm{C}$ (denaturation), $55^{\circ} \mathrm{C}$ for $1 \mathrm{~min}$ (annealing), $72^{\circ} \mathrm{C}$ for $1 \mathrm{~min}$ (elongation). Cycling was followed by a final extension step at $72^{\circ} \mathrm{C}$ for $10 \mathrm{~min}$. In a second step, PCR products were digested with the restriction enzyme BseR1 (New England Biolabs, Frankfurt, Germany) to further identify the amplified products for GAG deletion. The GAG deletion in $\triangle \mathrm{E}$-torsinA cDNA results in the elimination of a restriction site for the enzyme BseR1, which recognises the DNA sequence GAG/GAG. As an internal control, $\beta$-actin was analysed via PCR of genomic DNA from stool droplets or tail biopsies of the same animals by the following primer set: sense primer: 5'-CCACAGCTGAGAGGGAAATC-3' and antisense primer 5'-AGGCTGGAAAAGAGCCTCAG-3' in accordance with the PCR protocol described above. Reactions containing no template $\left(\mathrm{H}_{2} \mathrm{O}\right)$ were used to verify that obtained amplicons in the real-time PCR were not derived from contaminations. Analyses were done after electrophoresis on a gel containing $7 \%$ polyacrylamide by ethidium bromide staining.

\section{Results}

Whereas 21 day old mice usually excreted an average amount of 2 stool droplets within 1-2 minutes after placing them in a new and empty cage, 10 day old mice did not show spontaneous defecation. The analysis of DNA extracts of $200 \mathrm{mg}$ stool specimen from 21 day old mice provided a strong signal of the transgene (e.g. samples $\mathrm{G}$ and $\mathrm{H}$ extracted with the EURx ${ }^{\circledR}$ kit, Fig. 1b), comparable to that obtained with the tail biopsies

Fig. 2: Example of ethidium bromide stained gel electrophoresis of $\beta$-actin cDNA.

PCR amplification of genomic DNA extracted from stool samples or from tail biopsies yielded a $188 \mathrm{bp}$ DNA fragment (stool samples: $A-G$, tail biopsies: $H, I)$, independent of whether they were genotyped DYT1 positive $(A, C, D, G, H)$ or negative $(B, E$, $F, I)$. No differences in the signal intensity between the different commercial kits for stool analysis were observed. The negative control sample $\left(\mathrm{H}_{2} \mathrm{O}\right.$ without template) produced no signal $(\mathrm{J})$. (samples E and F in Fig. 1a). All three commercial kits resulted in a suitable detection of the transgene: PCR amplification of $\triangle \mathrm{E}$-torsinA cDNA out of stool and tail specimens yielded a 213 bp DNA product and digestion of PCR amplified product from $\triangle \mathrm{E}$-torsinA cDNA yielded DNA fragments of $95 \mathrm{bp}$ and $118 \mathrm{bp}$ due to the loss of a BseR1 restriction site in transgenic animals (Fig. 1a,b). These results were comparable to previous data of Shashidharan et al. (2005), who generated and initially described this transgenic mouse line. As shown in Figure 1a and 1 b, already a small amount of only 2 stool droplets $(\sim 45 \mathrm{mg})$, easily acquired by a short singular handling of the animals, was sufficient to gain an appropriate indication of the transgene in all three tested commercial kits. Although sufficient, the signal of the innuPREP was weaker after analysis of a small amount of stool sample compared to the two other kits (Fig. 1b) and maybe this result mirrors the producer's recommendation to use higher sample amounts for the innuPREP ${ }^{\circledR}$ kit (200-400 mg) in comparison to the two other kits (QIAamp ${ }^{\circledR}$ : $180-220$ mg; EURx ${ }^{\circledR}$ : $200 \mathrm{mg}$ ). As expected, genotyping of non-transgenic wildtype mice produced no signal in the PCR analysis of the DYT1 transgene, neither by using stool samples nor by using tail biopsies (Fig. 1a,b). The PCR analysis of $\beta$-actin DNA, done with genomic DNA which was extracted from stool samples and tail biopsies, revealed clear signals in formerly genotyped DYT1 positive and negative animals, whereas no signal was detected in the negative control sample $\left(\mathrm{H}_{2} \mathrm{O}\right.$ without template) (Fig. 2).

\section{Discussion}

The present results clearly show that the non-invasive genotyping method represents an effective modality that can replace invasive tissue sampling procedures currently used for the gen-

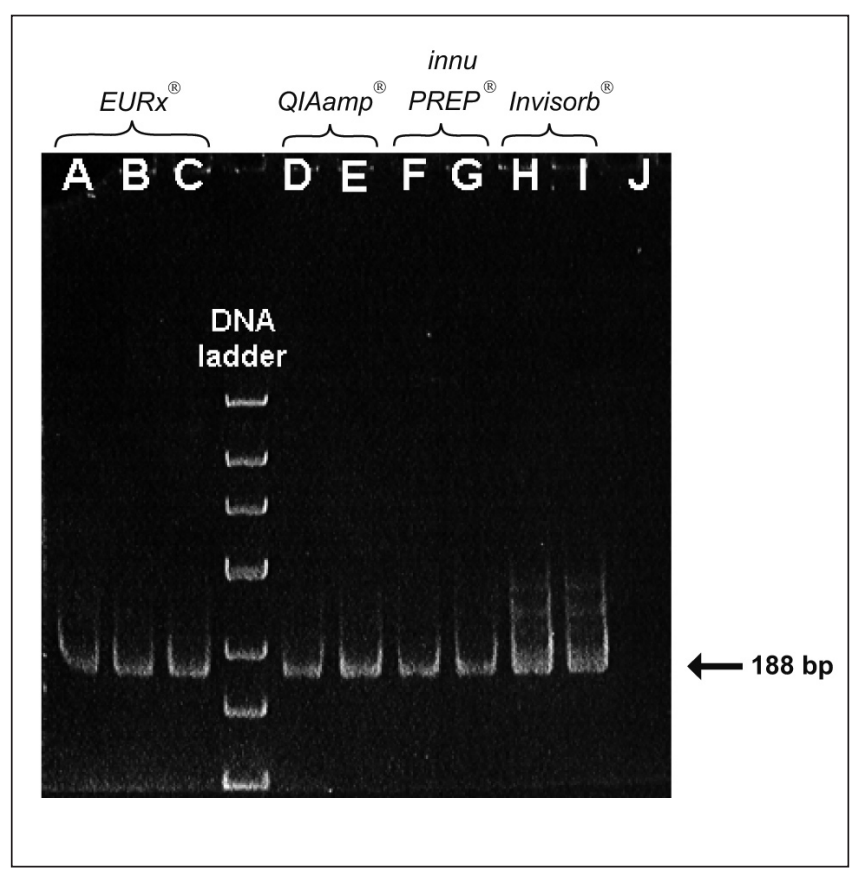


otyping of transgenic mice. Therefore, our results are in line with previous descriptions of stool specimen analysis as an adequate and reliable tool to detect the presence of transgenes and endogenous genes in mice (Broome et al., 1999; Kalippke et al., 2009; Murgatroyd et al., 2006). All three tested commercial kits were able to produce strong signals from stool specimens, which were comparable to those of tail biopsies. The detection of $\beta$-actin signals after DNA extraction and PCR analysis from stool samples also in DYT1 negative animals verified that the DNA extraction from stool samples itself was successful, supporting the suitability of this method. Nevertheless, a clear disadvantage of the method is the fact that it cannot be applied to 10 day old mice, since they do not spontaneously produce stool droplets after placing them in a new cage. However, it should be considered that the greatest part of genotyping on mice is performed at an age of 3-4 weeks.

Interestingly, also a small amount of stool droplets, sampled by singular placing of the animal in a new cage, was sufficient to produce a suitable signal. Although time or money should never be stated as reasons against animal welfare, one factor that is often declared to be a disadvantage and thereby a reason for the rare use of genotyping by stool samples is the fact that it requires more time to collect samples than for invasive methods, e.g. tail or ear biopsies (personal communications of the delegates for animal welfare of different laboratory animal facilities in Berlin, Germany). In fact, for stool amounts of $\sim 200 \mathrm{mg}$, recommended by the producers of the commercial kits, stool samples have to be collected three to four times per mouse; hence repeated handling is required, while tail or ear biopsies only require one handling procedure. The present data demonstrate that a small amount of $\sim 40 \mathrm{mg}$ was sufficient to detect the transgene. In 21 day old mice, this amount was usually excreted within 1-2 minutes, a time frame that is also required for tail or ear biopsy or blood sampling. Furthermore, several mice can be easily processed at the same time paint. Broome et al. (1999) did not detect any difference between the analysis of fresh stool or of stool that was up to $24 \mathrm{~h}$ old. Therefore, sampling of stool droplets does not need to be done mouse by mouse. Instead one mouse after the other can be placed quickly in a prepared clean, empty cage (one cage per mouse) without time-consuming fixation, and collection of stool droplets can be done later. This is a clear advantage in comparison to tail or ear biopsies, since fixation for these invasive methods allows handling of only one animal per time point. Nevertheless, it has to be mentioned that the need for cages is material consuming, a fact which may limit the use of this method in large laboratory animal facilities. As shown in the present study, the use of commercial kits, which include inhibitors of enzymes and impurities in faeces, simplifies the isolation and purification of genomic DNA from stool samples. Therefore, our results now clearly indicate that the non-invasive tissue sampling method presented here is also practical and does not require more time than invasive methods.

Normally, repeated sampling of a tail biopsy is not necessary. But although the sampling procedure, the extraction and the PCR protocols for genotyping are generally optimised, malfunction may occur in exceptional cases. Under these circumstances, the possibility of repeated sampling of stool samples represents a clear advantage in comparison to tail biopsies. Murine intestinal epithelia regenerate every 3-4 days (Chang and Leblond et al., 1971) and therefore "old" cells with genetic information enter the gut lumen and are excreted with the faeces. In case of inaccurate sampling, DNA extraction or PCR analysis, stool specimens can easily be resampled, while this would not be possible with tail biopsies. Although these cases are rare, this advantage may contribute to modestly reducing the number of genetically modified rodents. During the past years, several other alternatives of non-invasive genotyping methods were proposed, like sampling of saliva by oral swabs or pipettes, the use of hairs or of rectal cells sampled by rectal swabs (for review: Pinkert, 2003; Robinson et al., 2003; Schneider and Wolf, 2005). Although they represent non-invasive methods, they require restraining of animals and manipulations in the mouth, the rectum or on the fur of animals and therefore cause discomfort. In addition, methodological problems like the requirement of nested PCR in younger animals in case of saliva samples and a higher incidence of carrying over contaminations are presumed (Broome et al., 1999; Irwin et al., 1996; Robinson et al., 2003; Schneider and Wolf, 2005; Truett et al., 2000). Therefore, in view of its efficacy in refinement, genotyping via PCR analysis from stool droplets seems to be more suitable than other proposed non-invasive methods.

Admittedly, we cannot exclude the occurrence of false positive genotyping results by coprophagy or eating of other animal's hairs in the present study as previously found by Schubbert et al. (1994). Nevertheless, this appears very unlikely in the study described here, since we have genotyped positive and negative animals (littermates) which were housed together for 21 days. Therefore, one can presume that material of coprophagy is digested, i.e., destroyed by enzymes, within the intestines of animals and false positive results do not play a critical role.

Although meanwhile four studies, including the one presented here, have evidenced the efficacy of genotyping by DNA extraction from stool samples (Broome et al., 1999; Kalippke et al., 2009; Murgatroyd et al., 2006), this method cannot be used in general yet. Every specific gene must be tested for its suitability for this method since fragment size and origin of the gene may play a critical role. In view of the low number of tested gene constructs, the declaration that the method is a reproducible tool must be stated cautiously. Therefore, to get more consistent information about this method, it would be desirable that different laboratories with different PCR protocols test whether stool specimens represent an appropriate source of genomic DNA for their applications.

In the case of permanent marking of individual transgenic mice by ear-punching, the ear tissue can be used as a source of DNA for genotyping (Robinson et al., 2003) instead of stool droplets. However, it has been suggested to prefer noninvasive marking of laboratory rodents, like the use of marker pens or hair clipping (FELASA, Jennings et al., 1998; Robinson et al., 2003). Of course, the combination of a non-invasive marking method with a non-invasive tissue sampling method for genotyping would be ideal for refinement in genetically modified rodents. Nevertheless, it has to be taken into account 
that all non-invasive marking methods, like clipping patterns in the fur or marking the base of the tail or the fur with different colours, which can be easily and quickly done simply by grasping a mouse by its tail and without restraint, are only visible for 1-2 weeks. Although mice can be easily remarked when they are routinely transferred into a new home cage, this might be too labour intensive in large breeding facilities where only permanent invasive marking methods can assure the consistent identification of genotyped animals. But despite the fact that an invasive method has to be used for marking in these cases, one may consider whether a second invasive intervention, i.e. genotyping of animals by a tail biopsy, is necessary or may be replaced by non-invasive genotyping using stool samples.

In summary, the approach of non-invasive genotyping using stool samples and commercial kits represents a useful and practical tool in terms of animal welfare with regard to the " $3 \mathrm{R}$ concept", since it clearly refines the use of genetically modified mice by replacing painful invasive tissue sampling procedures. However, its suitability has been investigated for the extraction of only four specific gene constructs so far, including the data presented here (Broome et al., 1999; Kalippke et al., 2009; Murgatroyd et al., 2006). In addition, age and number of animals that must be genotyped have to be considered for adequate and practical use of the presented method. Therefore, this method can not be used in general so far and it would be desirable if scientists who work with genetically modified animals are encouraged to test whether this non-invasive method of tissue sampling can be used for determination of further gene constructs.

\section{References}

Arras, M., Rettich, A., Seifert, B. et al. (2007). Should laboratory mice be anaesthetized for tail biopsy? Lab. Anim. 41, $30-45$.

Breakefield, X. O., Blood, A. J., Li. Y. et al. (2008). The pathophysiological basis of dystonia. Nat. Rev. Neurosci. 9, 222234.

Broome, R. L., Feng, L., Zhou, Q. et al. (1999). Non-invasive mouse genotyping using stool analysis. FEBS Letters 462, 159-160.

Buehr, M., Hjorth, J. P., Hansen, A. K. et al. (2003). Genetically modified laboratory animals - what welfare problems do they face? J. Appl. Anim. Welf. Sci. 6(4), 319-338.

Chang, W. W. L. and Leblond, C. P. (1971). Renewal of the epithelium in the descending colon of the mouse. I. Presence of three cell populations: vacuolated-columnar, mucous and argentaffin. Am. J. Anat. 131, 73-99.

Irwin, M. H., Moffatt, J. and Pinkert, C. A. (1996). Identification of transgenic mice by PCR analysis of saliva. Nature Biotechnol. 14, 1146-1148.

Jennings, M., Batchelor, G. R., Brain, P. F. et al. (1998). Refining rodent husbandry: the mouse. Lab. Anim. 32, 233-259.

Kalippke, K., Werwitzke, S., von Hornung, M. et al. (2009).
DNA analysis from stool samples: a fast and reliable method avoiding invasive sampling methods in mouse models of bleeding disorders. Lab. Anim. 43, 390-393.

Murgatroyd, C., Bilko, D. and Spengler, D. (2006). Isolation of high-quality DNA for genotyping from faeces of rodents. Anal. Biochem. 348, 160-162.

Nicklas, W., Baneux, P., Boot, R. et al. (2002). Recommendations for the health monitoring of rodent and rabbit colonies in breeding and experimental units. Lab. Anim. 36, 20-42.

Pinkert, C. A. (2003). Transgenic animal technology: alternatives in genotyping and phenotyping. Comp. Med. 53 (2), 126-139.

Robinson, V., Morton, D. B., Anderson, D. et al. (2003). Refinement and reduction in production of genetically modified mice. Lab. Anim. 37, Suppl. 1, S1: 27-33.

Sauer, U. G., Kolar, R. and Rusche B. (2005). The use of transgenic animals in biomedical research in Germany. Part 1: Status Report 2001-2003. ALTEX 22 (4), 233-257.

Sauer, U. G., Kolar, R. and Rusche B. (2006). The use of transgenic animals in biomedical research in Germany. Part 2: Ethical evaluation of the use of transgenic animals in biomedical research and perspectives for the changeover in research to research animal-free methods. ALTEX 23 (1), 3-16.

Schneider, M. R. and Wolf, E. (2005). Genotyping of transgenic mice: Old principles and recent developments. Anal. Biochem. 344, 1-7.

Schubbert, R., Lettman, C. and Doerfler, W. (1994). Ingested foreign (phage M13) DNA survives transiently in the gastrointestinal tract and enters the bloodstream of mice. Mol. Gen. Genet. 242, 495-504.

Shashidharan, P., Sandu, D., Potla, U. et al. (2005). Transgenic mouse model of early-onset DYT1 dystonia. Hum. Mol. Genet. 14 (1), 125-133.

Tanabe, L. M., Kim, C. E., Alagem, N. et al. (2009). Primary dystonia: molecules and mechanisms. Nat. Rev. Neurol. 5, 598-609.

Truett, G. E., Heeger, P., Mynatt, R. L. et al. (2000). Preparation of PCR-quality mouse genomic DNA with hot sodium hydroxide and tris (HotSHOT). Biotechniques 29, 52-54.

\section{Acknowledgements}

We thank C. Kapfer for her excellent technical assistance and Dr. C. Gabler for providing the $\beta$-actin primers.

\section{Correspondence to}

Dr. M. Hamann

Institute of Pharmacology and Toxicology

Department of Veterinary Medicine

Freie Universität Berlin

Koserstr. 20, 14195 Berlin

Germany

e-mail: Hamann.Melanie@vetmed.fu-berlin.de 